\title{
Propriedades Psicométricas do Questionário de Adaptação ao Ensino Superior (QAES) em Estudantes Universitários Finalistas
}

\author{
Psychometric Properties of the Higher Education Adaptation Questionnaire (QAES) \\ in Finalist Undergraduates
}

\author{
Vanessa Domingues Ilha ${ }^{1}$, Acácia Aparecida Angeli dos Santos ${ }^{2}$ e \\ Francine Náthalie Ferraresi Rodrigues Queluz ${ }^{3}$
}

\begin{abstract}
Resumo
O Questionário de Adaptação ao Ensino Superior (QAES) busca identificar dificuldades de adaptação de graduandos. Em uma primeira versão, encontrou-se uma estrutura com 6 fatores. O objetivo deste estudo foi obter evidências de validade adicionais para o QAES. Para isso verificou-se as evidências baseadas na estrutura interna, precisão e evidências de validade baseadas nas relações com outras variáveis (adaptabilidade de carreira e autoeficácia). Participaram 406 universitários concluintes, idade média de 29,93 anos, que responderam ao QAES, à Escala de Autoeficácia na Formação Superior e à Escala de Adaptabilidade de carreira. A estrutura fatorial encontrada se ajustou à estrutura anterior (CFI=.96, RMSEA=.04; TLI=.95; $\left.x^{2} / \mathrm{gl}=1.79\right)$, apresentando medidas de precisão adequadas $(\alpha=.85)$. O QAES se relacionou com as medidas de adaptabilidade de carreira e autoeficácia. Estas medidas indicam que o instrumento apresenta evidências de validade interna e relacionado à outras variáveis.
\end{abstract}

Palavras-chave: autoeficácia, competência, satisfação profissional, engajamento

\begin{abstract}
The Higher Education Adaptation Questionnaire (QAES) seeks to identify undergraduate adaptation difficulties. In a first version, we found a structure with 6 factors. The aim of this study was to obtain additional validity evidence for QAES. For this purpose, we verified the evidence based on internal structure, reliability and validity evidence based on relationships with other variables (career adaptability and selfefficacy). Participated in this study 406 undergraduate students, mean age of 29.93 years, who answered the QAES, the Self-efficacy in Higher Education Scale and the Career Adaptability Scale. According to the results, the factorial structure fitted the previous structure (CFI=.96, RMSEA=.04; TLI=.95; $x 2 / \mathrm{gl}=1.79$ ), presenting adequate reliability outcomes $(\alpha=.85)$. The QAES was related to measures of career adaptability and self-efficacy. These measures indicate that the instrument presents internal validity and relationship to other variables.
\end{abstract}

Keywords: self-efficacy, competence, professional satisfaction, engagement

\footnotetext{
${ }^{1}$ Psicóloga e Mestre em Envelhecimento Humano, Docente na IMED e doutoranda em Psicologia na Universidade São Francisco. Rua Waldemar Cesar da Silveira, 105, Jardim Cura D'ars, Campinas - SP, Brasil. E-mail: vanessa.ilha @imed.edu.br

${ }^{2}$ Psicóloga, Mestre e Doutora em Psicologia, Professora Titular da Universidade São Francisco. Rua Waldemar Cesar da Silveira, 105, Jardim Cura D’ars, Campinas - SP, Brasil. E-mail: acácia.angeli@gmail.com

${ }^{3}$ Psicóloga, Mestre e Doutora em Psicologia, Pós-doutoranda em Psicologia na Universidade São Francisco. Rua Waldemar Cesar da Silveira, 105, Jardim Cura D’ars, Campinas - SP, Brasil. E-mail: francine.queluz@gmail.com

Revista Iberoamericana de Diagnóstico y Evaluación - e Avaliação Psicológica. RIDEP · No57 · Vol.4 · 41-51 · 2020

ISSN: 1135-3848 print /2183-6051online
} 


\section{Introdução}

Até recentemente o cenário do ensino superior no Brasil apresentava-se em expansão. As políticas de concessão de bolsas para alunos pouco privilegiados deram mais oportunidade de acesso ao ensino superior e com isso, ingressaram alunos em diferentes níveis de preparo formal na universidade. Em função de dificuldades não contempladas durante o ensino médio ou questões socioeconômicas, houve também o aumento no número de graduandos com dificuldades em se adaptar ao ensino superior (Wainer \& Melguizo, 2018). Somando-se ao ingresso na graduação, que por si só já exige capacidade de se adaptar a um novo contexto, podem surgir alguns problemas de adaptação ao ensino superior que são inerentes a esse novo processo, como por exemplo: desmotivação, falta de envolvimento com os colegas e com o curso (Tinto, 2007). De acordo com Tinto (2007), estar adaptado à universidade diz respeito à integração e à satisfação com a instituição, ser autônomo nas tomadas de decisões e cumprir com os compromissos acadêmicos.

O estudo realizado por Santos, Oliveira e Dias (2015), revelou que os docentes podem ser importantes para estimular os discentes a desenvolverem atividades em grupo, promovendo um ambiente mais saudável e facilitando as relações entre eles. Também, Ambiel e Barros (2018) verificaram que quanto maior a dificuldade em estabelecer vínculos na instituição, prejudicando a interação com os demais, maior será a probabilidade da desistência do curso pelo aluno. Ainda conforme os autores, estudantes mais satisfeitos com a escolha, estão mais aptos a serem mais organizados e adaptados à nova etapa. Percebe-se, então, que estudar como se dá a adaptação às exigências que um curso superior requer, envolve fatores que compreendem relações interpessoais expressas em amizades com pessoas do próprio curso assim como manter vínculos afetivos com eles (Soares et al., 2018).

Os estudantes se deparam com a realidade acadêmica, estando o processo de adaptação interligado à capacidade de conquistar novas amizades e superar a ansiedade e o estresse, característicos desta etapa (Luca, Noronha, \& Queluz, 2018). A decisão em permanecer ou não no curso acontece, também, a partir da percepção do aluno sobre seus relacionamentos interpessoais e na manutenção de bons relacionamentos, com professores, funcionários e colegas, além da adequação ao que é exigido pela instituição e aos recursos oferecidos por ela (Ambiel, Santos, \& Dalbosco, 2016; Granado, Santos, Almeida, Soares, \& Guisande, 2005; Santos et al., 2015).

Acreditar na capacidade de adaptação e pensar em um projeto de carreira, podem ser importantes preditores de sucesso na conclusão do curso superior (Santos et al., 2015). Outros aspectos que devem ser destacados estão relacionados ao estilo do aluno e sua característica de aprender (Oliveira, Santos, \& Inácio, 2018). Para mensurar a adaptação dos alunos ao contexto acadêmico e poder identificar as ações que possibilitariam o auxílio a esta permanência, foi utilizado um instrumento construído em parceria de estudiosos portugueses e brasileiros, qual seja, o Questionário de Adaptação ao Ensino Superior (QAES). A primeira versão elaborada por Araújo et al. (2014) foi avaliada com uma amostra de estudantes portugueses. Sendo encontrada uma estrutura fatorial composta por cinco fatores (projeto de carreira, adaptação social, adaptação pessoal-emocional, adaptação ao estudo e adaptação institucional) constituídos por oito itens cada um.

Mais recentemente, o estudo realizado por Dalbosco (2018) avaliou o quanto os construtos de autoeficácia e de autorregulação da aprendizagem estavam relacionados à adaptação ao ensino superior, utilizando a versão de Araújo et. al (2014) do QAES. Os participantes foram 404 graduandos iniciantes no Ensino Superior. A maioria da amostra era do sexo feminino $(n=$ 235). Da amostra total, aproximadamente 100 alunos apresentaram baixos escores no QAES (até 141 pontos). De acordo com os resultados da pesquisa, houve uma correlação alta entre a dimensão adaptação ao estudo (QAES) com a dimensão acadêmica do construto da autoeficácia, mostrando que a convergência entre estas dimensões das duas medidas confirma a hipótese inicial do estudo.

Importante lembrar que a autoeficácia se refere às crenças de uma pessoa na sua capacidade de executar tarefas (Bandura, 1993). No contexto acadêmico, autoeficácia diz respeito à capacidade do aluno para realizar e organizar as tarefas 
solicitadas pelos professores (Polydoro \& Guerreiro-Casanova, 2010). Paralelamente à importância de avaliar o construto da adaptação, é relevante mensurar a autoeficácia do estudante universitário e suas crenças acerca de suas capacidades para buscar resultados positivos. Ambos são construtos relacionados, pois como já dito anteriormente, alunos que conseguem se adaptar melhor também apresentam melhor percepção de autoeficácia para realizar as tarefas acadêmicas, assim como melhor satisfação com a vida (Dalbosco, 2018; Ornelas, Díaz-Leal, Contreras, Fernández, \& Pinto, 2019).

No âmbito da Teoria Social Cognitiva (TSC), Bandura (1993) esclarece que a autoeficácia diz respeito ao julgamento das pessoas sobre suas capacidades para organizar e executar cursos de ação necessários para alcançar certos tipos de desempenho. As crenças nas próprias capacidades frente à transição para o mercado de trabalho foi o propósito da pesquisa de Silva, Ferraz e Oswaldo (2012). Os autores entrevistaram 56 estudantes do oitavo e décimo semestre de três cursos (Pedagogia, Administração de Empresas e Psicologia) de uma Faculdade de Ensino Superior privada. Os resultados indicaram a existência de uma percepção da autoeficácia moderada dos formandos participantes da pesquisa, com pequena superioridade da dimensão adaptação ao trabalho. Os participantes com idade superior a 30 anos apresentaram valores maiores que os demais na dimensão adaptação ao trabalho. Em suma, o estudo verificou que mesmo depois de alguns anos no curso escolhido, os estudantes ainda não se sentem capazes de atuar no mercado de trabalho. Isto pode afetar, por exemplo, sua adaptabilidade para o exercício da carreira escolhida. A adaptabilidade de carreira também é um construto que, de acordo com a literatura, se relaciona com a adaptação ao ensino superior.

Proposta por Savickas et al., (2009), a adaptabilidade de carreira é composta por quatro dimensões: preocupação, controle, curiosidade e confiança, que podem ser entendidas como um conjunto de atitudes frente às demandas de carreira, fundamentais para o desenvolvimento adequado. Além disso, ela é concebida como a prontidão para lidar com tarefas previsíveis de preparação e participação em papéis e ajustes imprevisíveis impostos por mudanças nas condições de trabalho. No que se refere à dimensão preocupação, pessoas que não apresentam atitudes relacionadas a esta dimensão mostram-se apáticas quanto ao interesse e planejamento do futuro profissional. Este estado de não-preocupação, implica ausência de metas e planos, podendo estar associada a uma visão negativa e pessimista do futuro.

Falhas na dimensão controle geram indefinição, procrastinação, indecisão vocacional e um baixo engajamento da pessoa no processo de construção de sua carreira. Déficits na dimensão de curiosidade repercutem em posturas ingênuas, pobreza em exploração, pouco conhecimento da realidade e de si mesmo, e acarretam decisões de carreira pouco consistentes e uma visão limitada e talvez ilusória do mundo do trabalho. Por fim, os déficits de confiança produzem no indivíduo dificuldade para agir, por não se sentir capaz de realizar seus projetos de vida (Savickas, et al., 2009).

Assim como a adaptação ao ensino superior, a adaptabilidade de carreira tem relevância para o entendimento das características que prejudicam o sucesso acadêmico. Mognon e Santos (2013) estudaram a relação entre as vivências acadêmicas e o desenvolvimento de carreira com 208 universitários de duas instituições particulares, uma no estado de São Paulo $(n=133)$ e outra no estado de Minas Gerais $(n=75)$. As idades variaram entre 18 e 51 anos $(\mathrm{M}=23,53$; $\mathrm{DP}=7,49)$, sendo $62.5 \%$ do feminino. Os achados indicaram que o desenvolvimento de carreira estava relacionado ao planejamento de metas em relação à profissão, ao bem-estar físico, emocional e psicológico do estudante. Também sua estabilidade emocional, otimismo e autoconfiança são resultados das dimensões pessoal e de carreira, que se correlacionaram entre si de forma positiva. Outro resultado importante diz respeito ao estabelecimento de amizades e à ação de procurar a ajuda de professores ou colegas, como indicativo da crença do estudante de que seu sucesso profissional depende muito mais de seu esforço pessoal do que das circunstâncias ambientais (construto diretamente ligado à crença de autoeficácia acadêmica).

Os achados de Mognon e Santos (2013) indicaram que o desenvolvimento de carreira estava relacionado ao planejamento de metas em 
relação à profissão, ao bem-estar físico, emocional e psicológico do estudante. Também sua estabilidade emocional, otimismo e autoconfiança são resultados das dimensões pessoal e de carreira que se correlacionam entre si de forma positiva. Outro resultado importante diz respeito ao estabelecimento de amizades e a ação em procurar a ajuda de professores ou colegas, como indicativo da crença do estudante de que seu sucesso profissional depende muito mais de seu esforço pessoal do que das circunstâncias ambientais.

No mesmo sentido, Ambiel et al. (2016) realizaram um estudo para avaliar as relações entre as vivências acadêmicas, a adaptabilidade de carreira e os motivos para evasão. Alguns dos dados obtidos sugerem que as expectativas que os estudantes têm sobre o futuro de sua carreira, podem estar bastante relacionadas com as decisões de permanência ou não no curso. Anteriormente, Gamboa, Paixão e Palma (2014) analisaram o impacto da adaptabilidade de carreira na autoeficácia para a transição para o trabalho, considerando o efeito da empregabilidade percebida. A pesquisa foi realizada com 261 alunos do $1^{\circ}$ ciclo do ensino superior. A maioria foi do sexo feminino $(n=210$; $80.5 \%$ ), sendo que as suas idades se distribuíram entre os 17 e os 57 anos $(\mathrm{M}=22.53$; $\mathrm{DP}=6.77)$. $\mathrm{O}$ rendimento acadêmico (determinado pelo cálculo da média aritmética das classificações das unidades curriculares já concluídas até a data da participação no estudo) variou entre dez e 18 valores $(M=13.99 ; \mathrm{DP}=.83)$, em uma escala de 20 valores. Os instrumentos utilizados foram questionário sociodemográfico que solicitava informação relativa à idade, sexo, curso, rendimento escolar e satisfação com o curso, e a Escala de Adaptabilidade de Carreira. Estudantes com maiores níveis de adaptabilidade se mostraram mais confiantes na resolução de tarefas relativas à transição para o trabalho.

Considerando os resultados já mencionados, ressalta-se a importância do investimento, por parte das IES, em estratégias para adaptar o estudante ao contexto do ensino superior. Para tanto, o QAES mostrou ser um instrumento com bons parâmetros psicométricos e seria importante verificar se ele está relacionado também aos construtos autoeficácia e adaptabilidade de carreira. Aferir e ter medidas confiáveis para verificar o quanto um aluno está adaptado ao ensino superior se torna altamente relevante, ao se pensar no que pode ser realizado com esta informação, como por exemplo, planejar intervenções que melhorem a forma como se dá a entrada e contribuir para a permanência no ensino superior.

Diante disso, o objetivo desta pesquisa foi obter evidências de validade adicionais para o QAES. Para isso, verificou-se as evidências de validade baseadas na estrutura interna, as estimativas de precisão e as evidências de validade baseada nas relações com outras variáveis (adaptabilidade de carreira e autoeficácia). Como hipótese norteadora, esperava-se que a estrutura fatorial encontrada por Araújo et al. (2014) se ajustasse a da amostra deste estudo, com bons índices de precisão e que o instrumento apresentasse correlação positiva com autoeficácia e adaptabilidade de carreira.

\section{Método}

\section{Participantes}

A amostra foi constituída por 406 estudantes universitários, 149 (36.6\%) do sexo masculino e 257 (63.4\%) do sexo feminino, com média de idade de $29.93(D P=5.51)$ anos, todos concluintes de curso. Para esta pesquisa considerou-se como concluinte $o$ aluno que frequentava a graduação a partir do oitavo semestre. Os cursos envolvidos foram: Psicologia, Sistemas de Informação, Direito, Administração, Odontologia, Arquitetura e Engenharia Civil, de uma instituição de ensino superior do interior do estado Rio Grande do Sul, Brasil. Os critérios para participação do aluno incluíram estar devidamente matriculado e frequentando os semestres finais a partir do oitavo período e não participaram da pesquisa alunos que não fossem concluintes, mesmo que estivessem cursando a mesma disciplina dos demais alunos finalistas.

\section{Questionário sociodemográfico}

Elaborado para a caracterização da amostra. As questões incluíram itens para a identificação, idade, sexo e curso. 
Questionário de Adaptação ao Ensino Superior (QAES), Araújo et al. (2014)

Trata-se de um instrumento de autorrelato que pretende avaliar as vivências, opiniões e sentimentos com relação à adaptação acadêmica ao Ensino Superior. A escala é comporta por 40 itens distribuídos em cinco dimensões: projeto de carreira (Exemplo: "estou no curso superior que sempre sonhei"), adaptação social (Exemplo: "faço amigos com facilidade nesta universidade"), adaptação pessoal-emocional (Exemplo: "nas últimas semanas tenho me sentido ansioso"), adaptação ao estudo (Exemplo: "planejo diariamente as minhas atividades de estudo") $e$ adaptação institucional (Exemplo: "tenho bons professores na minha universidade") apresentados em formato Likert de 1(discordo totalmente) até 5 (concordo totalmente). A pontuação total possível é de 200 pontos, caso o respondente manifeste concordância total em todos os itens. Estudos iniciais com o instrumento mostraram a existência de evidências de validade pela análise da estrutura interna dos itens, que aponta para uma configuração de seis fatores, a saber, curso $(\alpha=.92)$, interpessoal $(\alpha=.92)$, pessoal-emocional $(\alpha=.91)$, acadêmica $(\alpha=.83)$, instituição $(\alpha=.72) \mathrm{e}$ carreira $(\alpha=.82)$. A consistência interna dos itens variou de .72 a .92 para as diferentes dimensões, $o$ que indica estimativas favoráveis de precisão.

\section{Escala de Autoeficácia na Formação Superior} (AEFS), Polydoro e Guerreiro-Casanova (2010)

É um instrumento de autorrelato que objetiva identificar a autoeficácia de estudantes em organizar e executar cursos de ações requeridos para produzir certas realizações referentes aos aspectos compreendidos pelas tarefas acadêmicas pertinentes ao curso superior. A escala tem 58 itens, em formato Likert de 1 (pouco capaz) até 10 (muito capaz), divididos em cinco dimensões: autoeficácia acadêmica (Exemplo: "Quanto eu sou capaz de entender as exigências do meu curso?"), autoeficácia na regulação da formação (Exemplo: "Quanto eu sou capaz de tomar decisões relacionadas à minha formação?”), autoeficácia na interação social (Exemplo: "Quanto eu sou capaz de trabalhar em grupo?”), autoeficácia em ações pró-ativas (Exemplo: "Quanto eu sou capaz de contribuir com ideias para a melhoria do meu curso?"), autoeficácia na gestão acadêmica
(Exemplo: "Quanto sou capaz de motivar-me para fazer as atividades ligadas ao meu curso?"). A pontuação máxima a ser obtida é de 580 pontos. A evidência de validade obtida para o instrumento, foi baseada na análise da estrutura interna dos itens, que mostrou a existência de cinco fatores, a saber: autoeficácia $(\alpha=.88)$, autoeficácia na regulação da formação apresenta $(\alpha=.87)$, autoeficácia na interação social $(\alpha=.80)$, autoeficácia em ações pró-ativas $(\alpha=.85)$ e autoeficácia na gestão acadêmica $(\alpha=.80)$. O índice total para autoeficácia na Formação Superior foi .94 .

\section{Escala de Adaptabilidade de Carreira (EAC), Audibert e Teixeira (2015)}

Refere-se a um instrumento de autorrelato que avalia a capacidade que o sujeito tem de lidar com as transições profissionais. A escala é composta por 24 itens, distribuídos em quatro dimensões: preocupação (Exemplo: "Pensar sobre como será meu futuro"), controle (Exemplo: "Acreditar na minha capacidade de dirigir a própria vida"), curiosidade (Exemplo: "Explorar o ambiente ao meu redor") e confiança (Exemplo: "Superar obstáculos") e resultou da tradução e adaptação da versão em inglês (Career Adapt-Abilities Scale, Savickas, \& Porfeli, 2012). O estudo de evidências de validade ocorreu pela análise da estrutura interna dos itens, que foram agrupados em quatro dimensões. A fidedignidade do instrumento foi avaliada por meio do alfa total .94 e os índices das dimensões foram: preocupação $(\alpha=.78)$, controle $(\alpha=.89)$, curiosidade $(\alpha=.87)$, confiança $(\alpha=.83)$.

\section{Procedimento de coleta de dados}

Inicialmente, foi solicitada a autorização para a coleta de dados junto à Instituição de Ensino Superior, que foi anexada ao projeto quando enviado ao Comitê de Ética em Pesquisa da Universidade São Francisco, aprovado sob número CAAE: 53659816.5.0000.5514. Após a aprovação, foi combinado previamente com o professor de cada turma da instituição, a coleta de dados, em sala de aula. Todos os alunos que optaram por participar da pesquisa, assinaram o Termo de Consentimento Livre e Esclarecido (TCLE). Em seguida foi entregue o questionário sociodemográfico, o QAES, a Escala de 
Autoeficácia para Formação Superior, e a Escala Adaptabilidade de Carreira. Os participantes responderam nesta ordem.

\section{Procedimento de Análise de dados}

Para verificar a distribuição dos escores para cada instrumento, foram calculadas a média, mediana, desvio padrão, os valores mínimos e máximos, além de indicadores de curtose e assimetria para cada variável. As análises descritivas foram realizadas pelo Statistical Package for the Social Sciences (SPSS). Todas as variáveis apresentaram uma distribuição normal, segundo a inspeção do número de modas, valores de curtose e assimetria e do teste de normalidade de Kolmogorov-Smirnov (Ledesma, Ferrando, \& Tosi, 2019; Marôco, 2014; Pasquali, 2015).

Para testar o modelo proposto por Araújo et al. (2014) foi realizada uma análise fatorial confirmatória via structural equation modeling, isto é, inferindo que os construtos do modelo se relacionam entre si (Schreiber, Nora, Stage, Barlow, \& King, 2006) no software MPLUS. Os índices de ajuste considerados foram: Comparative Fit Index (CFI $\geq .90)$, Root Mean Square Error of Approximation (RMSEA, $\leq .06$ ou $\leq .08$; com intervalo de confiança de $90 \%$ ), o Tucker-Lewis Index (TLI Z.95), a significância estatística do teste de Qui-quadrado ( $p \leq .05)$ e o Qui-quadrado dividido pelo grau de liberdade $\left(x^{2} / \mathrm{gl}<3\right)$ (Hu \& Bentler, 1999). Para verificar possíveis correlações, foram realizadas análises por meio do teste de correlações de Pearson, uma vez que a amostra apresentou uma distribuição normal. Estas medidas foram calculadas por meio do SPSS. Para o presente estudo, a magnitude das correlações foi classificada em: fraca $(<.30)$, moderada (.30 a .59$)$, forte (.60 a .99) ou perfeita $(1,0)$ (Levin \& Fox, 2004). Para avaliar a confiabilidade do instrumento, foi utilizado o cálculo do alfa de Cronbach.

\section{Resultados}

Os resultados da análise fatorial confirmatória (AFC) realizada com os dados dos alunos finalistas de curso constam no Quadro 1. Nela serão apresentados os índices de ajuste referentes ao modelo de Araújo et al. (2014) constituído por cinco fatores.
Quadro 1. Índices de ajuste do modelo do QAES proposto por Araújo et al. (2014) testados na AFC

\begin{tabular}{lccccc}
\hline Modelo & $x^{2} / \mathrm{gl}$ & $p$ & CFI & RMSEA & TLI \\
\hline $\begin{array}{l}\text { Valor de } \\
\text { referência }\end{array}$ & $<3$ & $<.05$ & $\geq .90$ & $<.08$ & $\geq .95$ \\
$\begin{array}{l}\text { Cinco } \\
\text { Fatores }\end{array}$ & 1.79 & .001 & .96 & .04 & .95 \\
\hline
\end{tabular}

De acordo com os resultados observados no Quadro 1, todos os índices de ajuste se mostraram adequados, exceto o valor de $p$. No entanto, o fato de ele ser significativo pode ser em decorrência do tamanho da amostra. Considerando que todos os outros índices de ajuste se mostraram adequados pode-se afirmar que a estrutura fatorial obtida anteriormente foi confirmada (Hu \& Bentler, 1999). No Quadro 2 são apresentados os índices de correlação, obtidos entre o escore total do QAES e seus respectivos fatores.

Nos resultados do Quadro 2 observa-se que todas correlações foram significativas e que as de maior magnitude foram entre o escore total do QAES e os escores de projeto de carreira, adaptação social, adaptação ao estudo e adaptação institucional e magnitude forte. As menores correlações foram entre $\mathrm{o}$ fator adaptação pessoal-emocional com o escore total e com os fatores adaptação social, adaptação ao estudo e adaptação institucional que foram de magnitude fraca. Todas as outras correlações foram de magnitude moderada.

Os valores de precisão obtidos no presente estudo foram: projeto de carreira $\alpha=.89$; adaptação social, $\alpha=.88$; adaptação pessoalemocional, $\alpha=.86$; adaptação ao estudo $\alpha=.79$ e adaptação institucional $\alpha=.80$. Todos são considerados valores adequados (Tornimbeni, Pérez, \& Olaz, 2008). O alfa de Cronbach $(\alpha=.85)$ geral foi excelente. No que diz respeito à carga fatorial, na amostra do presente estudo, todos os itens apresentaram carga fatorial acima de .40. Assim, pode-se afirmar que eles representam bem o construto avaliado. 
Quadro 2. Correlações entre o Escore Total do QAES e os Escores nos Cinco Fatores

\begin{tabular}{|c|c|c|c|c|c|c|}
\hline & $\begin{array}{c}\text { Escore } \\
\text { total }\end{array}$ & $\begin{array}{c}\text { Fator } 1 \\
\text { Projeto de } \\
\text { carreira } \\
\end{array}$ & $\begin{array}{c}\text { Fator } 2 \\
\text { Adaptação } \\
\text { social } \\
\end{array}$ & $\begin{array}{c}\text { Fator } 3 \\
\text { Adaptação pessoal- } \\
\text { emocional }\end{array}$ & $\begin{array}{c}\text { Fator } 4 \\
\text { Adaptação ao } \\
\text { estudo } \\
\end{array}$ & $\begin{array}{c}\text { Fator } 5 \\
\text { Adaptação } \\
\text { institucional } \\
\end{array}$ \\
\hline Fator 1 & $.67 * *$ & & & & & \\
\hline Fator 2 & $.63 * *$ & $.33 * *$ & & & & \\
\hline Fator 3 & $.14^{*}$ & $.33 * *$ & $.19 * *$ & & & \\
\hline Fator 4 & $.65^{* *}$ & $.49 * *$ & $.36^{* *}$ & $.24 * *$ & & \\
\hline Fator 5 & $.71 * *$ & $.44 * *$ & $.37 * *$ & $.11 * *$ & $.39 * *$ & - \\
\hline
\end{tabular}

Quadro 3. Saturação dos Itens do QAES por Fator e seus valores de confiabilidade

\begin{tabular}{|c|c|}
\hline Fator/Itens & Saturação \\
\hline Fator 1 - Projeto de carreira & $\alpha=.89$ \\
\hline Item 5 & .78 \\
\hline Item 10 & .75 \\
\hline Item 15 & .75 \\
\hline Item 20 & .44 \\
\hline Item 25 & .77 \\
\hline Item 30 & .87 \\
\hline Item 35 & .81 \\
\hline Item 40 & .72 \\
\hline Fator 2 - Adaptação social & $\alpha=.88$ \\
\hline Item 2 & .60 \\
\hline Item 7 & .85 \\
\hline Item 12 & .86 \\
\hline Item 17 & .70 \\
\hline Item 22 & .68 \\
\hline Item 27 & .66 \\
\hline Item 32 & .81 \\
\hline Item 37 & .79 \\
\hline Fator 3 - Adaptação pessoal-emocional & $\alpha=.86$ \\
\hline Item 4 & .57 \\
\hline Item 9 & .73 \\
\hline Item 14 & .73 \\
\hline Item 19 & .74 \\
\hline Item 24 & .82 \\
\hline Item 29 & .54 \\
\hline Item 34 & .74 \\
\hline Item 39 & .76 \\
\hline Fator 4 - Adaptação ao estudo & $\alpha=.79$ \\
\hline Item 3 & .52 \\
\hline Item 8 & .48 \\
\hline Item 13 & .62 \\
\hline Item 18 & .78 \\
\hline Item 23 & .54 \\
\hline Item 28 & .75 \\
\hline Item 33 & .65 \\
\hline Item 38 & .37 \\
\hline Fator 5 - Adaptação institucional & $\alpha=.80$ \\
\hline Item 1 & .71 \\
\hline Item 6 & .58 \\
\hline Item 11 & .49 \\
\hline Item 16 & .51 \\
\hline Item 21 & .64 \\
\hline Item 26 & .54 \\
\hline Item 31 & .64 \\
\hline \multirow[t]{2}{*}{ Item 36} & .61 \\
\hline & $\begin{array}{c}\alpha \\
\text { total=.85 }\end{array}$ \\
\hline
\end{tabular}

Evidências de validade baseada nas relações com outras medidas que avaliam construtos relacionados

No Quadro 4 apresenta-se as correlações entre os fatores do QAES e cada um dos fatores da Escala de Autoeficácia para a Formação Superior (AEFS). Também, foram calculadas as correlações entre os fatores do QAES e os da Escala de Adaptabilidade de Carreira (EAC).

Percebe-se que todas as correlações foram estatisticamente significativas. As maiores correlações foram entre adaptação ao estudo do QAES e autoeficácia acadêmica e autoeficácia na gestão acadêmica da EAFS, assim como com seu escore total, sendo correlações de magnitude forte. Já as menores correlações foram entre a adaptação pessoal-emocional do QAES e a autoeficácia na gestão acadêmica da EAFS, também entre adaptação social QAES e todas as medidas da EAC (preocupação, controle, curiosidade e confiança). $\mathrm{Da}$ mesma forma as correlações foram menores entre a adaptação pessoal-emocional do QAES e a autoeficácia na interação social da EASF, a adaptação ao estudo e adaptação institucional da EAFS, e entre adaptação pessoal-emocional do QAES e a preocupação, o controle e a curiosidade da EAC. Ainda foram menores as correlações entre a adaptação institucional do QAES e a autoeficácia na interação social da EAFS, a autoeficácia em ações proativas e a autoeficácia na gestão acadêmica, e por fim, entre adaptação institucional do QAES e a curiosidade e a confiança da EAC.

Todas estas correlações foram de magnitude fraca. As demais correlações encontradas foram de magnitude moderada. Ademais, ao comparar os dois instrumentos avaliados, para verificar as evidências de validade baseadas na relação com outras variáveis, percebe-se que as correlações, de modo geral, foram de maior magnitude entre as medidas do QAES com as medidas de autoeficácia. 
Quadro 4. Correlações entre Escores do QAES com os Escores Totais e com os fatores da EAFS e da EAC

\begin{tabular}{|c|c|c|c|c|c|c|}
\hline & $\begin{array}{c}\text { Escore Total } \\
\text { QAES }\end{array}$ & $\begin{array}{l}\text { Fator } 1 \\
\text { Projeto de } \\
\text { carreira }\end{array}$ & $\begin{array}{c}\text { Fator } 2 \\
\text { Adaptação } \\
\text { social }\end{array}$ & $\begin{array}{c}\text { Fator } 3 \\
\text { Adaptação } \\
\text { pessoal- } \\
\text { emocional }\end{array}$ & $\begin{array}{c}\text { Fator } 4 \\
\text { Adaptação ao } \\
\text { estudo }\end{array}$ & $\begin{array}{c}\text { Fator } 5 \\
\text { Adaptação } \\
\text { institucional }\end{array}$ \\
\hline EAFS Total & $.48^{*}$ & $.50^{*}$ & $.36^{*}$ & $.35^{*}$ & $.64^{*}$ & $.31 *$ \\
\hline $\begin{array}{l}\text { EAFS - F1 } \\
\text { Autoeficácia acadêmica } \\
\text { EAFS - F2 }\end{array}$ & $.43^{*}$ & $.48^{*}$ & $.29 *$ & $.35^{*}$ & $.60^{*}$ & $.30 *$ \\
\hline $\begin{array}{l}\text { Autoeficácia na regulação da } \\
\text { formação }\end{array}$ & $.43^{*}$ & $.50 *$ & $.31 *$ & $.34 *$ & $.55^{*}$ & $.31 *$ \\
\hline $\begin{array}{l}\text { EAFS - F3 } \\
\text { Autoeficácia na interação social }\end{array}$ & $.43^{*}$ & $.36^{*}$ & $.45^{*}$ & $.23 *$ & $.45^{*}$ & $.24^{*}$ \\
\hline $\begin{array}{l}\text { EAFS - F4 Autoeficácia em ações } \\
\text { proativas }\end{array}$ & $.45^{*}$ & $.45^{*}$ & $.32 *$ & $.29 *$ & $.58^{*}$ & $.28 *$ \\
\hline $\begin{array}{l}\text { EAFS - F5 Autoeficácia na gestão } \\
\text { acadêmica }\end{array}$ & $.42 *$ & $.42 *$ & $.26^{*}$ & $.28^{*}$ & $.63^{*}$ & $.28^{*}$ \\
\hline EAC Total & $.38^{*}$ & $.43^{*}$ & $.28^{*}$ & $.32 *$ & $.45^{*}$ & $.32 *$ \\
\hline $\begin{array}{l}\text { EAC F1- } \\
\text { Preocupação }\end{array}$ & $.42 *$ & $.44^{*}$ & $.25^{*}$ & $.26^{*}$ & $.43^{*}$ & $.34^{*}$ \\
\hline $\begin{array}{l}\text { EAC F2 - } \\
\text { Controle }\end{array}$ & $.33^{*}$ & $.33^{*}$ & $.27^{*}$ & $.26^{*}$ & $.40^{*}$ & $.30^{*}$ \\
\hline $\begin{array}{l}\text { EAC F3 - } \\
\text { Curiosidade }\end{array}$ & $.30 *$ & $.34^{*}$ & $.24 *$ & $.26^{*}$ & $.38^{*}$ & $.22 *$ \\
\hline $\begin{array}{l}\text { EAC F4 - } \\
\text { Confiança }\end{array}$ & $.29 *$ & $.44 *$ & $.22 *$ & $.31 *$ & $.36^{*}$ & $.25^{*}$ \\
\hline
\end{tabular}

\section{Discussão}

Esta pesquisa foi realizada com o objetivo de obter evidências de validade adicionais para o QAES. Para isso, verificou-se as evidências de validade baseadas na estrutura interna do QAES, as estimativas de precisão e as evidências de validade baseada nas relações com outras variáveis: a adaptabilidade de carreira e autoeficácia. $\mathrm{Na}$ análise da estrutura interna, os resultados obtidos no QAES para alunos concluintes, vão ao encontro do modelo proposto por Araújo et al. (2014), que apresentou uma medida de avaliação, com cinco dimensões, confirmando a hipótese inicial. Com relação à carga fatorial, na amostra deste estudo, todos os itens apresentaram carga fatorial acima de .40, possibilitando afirmar que eles representam bem à adaptação ao ensino superior, construto avaliado.

No que diz respeito às evidências de validade baseadas na relação com outras variáveis, percebe-se que todas as correlações foram estatisticamente significativas, confirmando também as hipóteses iniciais e corroborando outros estudos existentes (Bardagi \& Hutz, 2011; Gamboa et al., 2014). As maiores correlações foram entre o fator adaptação ao estudo do QAES e os fatores autoeficácia acadêmica e autoeficácia na gestão acadêmica, assim como seu escore total sendo estas correlações de magnitude forte. Estes resultados condizem com os apresentados por vários pesquisadores (Bardagi \& Hutz, 2011; Costa, Araújo, \& Almeida, 2014; Mognon \& Santos, 2013; Rosas, 2013). A hipótese de que estão mais adaptados alunos que creem nas suas capacidades de resolução de problemas e confiam na sua capacidade de aprender e demonstrar o conteúdo aprendido, se confirmou (Luca et al., 2018). Nesse mesmo sentido, o estudo de Ambiel e Barros (2018) defendem que quanto mais adaptado emocionalmente e socialmente à instituição, menores são as possibilidades de abandono do curso. Os resultados alcançados por Dalbosco (2018) mostram também que alunos com bons índices de adaptação pessoal-emocional possuem maiores níveis de competência. Além disso, estudantes que constroem um projeto de carreira, também parecem estar mais adaptados. A confirmação da hipótese da relação da autoeficácia com a adaptação ao ensino superior, corrobora o que é encontrado na literatura (Alliprandim \& Souza 2016; Gamboa et al., 2014).

As correlações entre os escores do QAES com os escores totais e com os fatores da EAFS e da EAC, foram estatisticamente significativas, em 
que a autoeficácia na gestão acadêmica da EAFS obteve uma correlação forte com a adaptação ao estudo do QAES, que permite inferir que a confiança percebida pelo estudante, na sua capacidade de envolver-se nas atividades acadêmicas bem como planejá-las e cumprir os prazos determinados, são indícios de sucesso na adaptação ao ensino superior (Marôco, 2014). Também foi forte a correlação dos itens do QAES com seu escore total, inferindo que aqueles alunos que se percebem mais autoeficazes, obtém sucesso na adaptação acadêmica.

As correlações entre os fatores adaptação social do QAES e o fator autoeficácia na gestão acadêmica da EAFS, foram fracas e permitem afirmar que alunos que não conseguem aproximação com o grupo de colegas, e não se adaptam ao ambiente universitário, tendem a encontrar dificuldades na adaptação. Conforme Stajkovi, Bandura, Lockec, Lee e Sergent (2018), aqueles alunos com mais estabilidade emocional, e cujos pais e professores auxiliam na promoção do aprendizado, tendem a ter mais crenças nas suas capacidades. Também, aqueles alunos que são mais confiantes em sua capacidade de aprender, em demonstrar e aplicar o conteúdo, aproveitam as oportunidades de formação e atualizam seus conhecimentos, se adaptam melhor ao contexto universitário (Alliprandini \& Souza, 2016; Soares, Seabra, \& Gomes, 2014).

\section{Conclusões}

Com relação os objetivos do presente estudo, conclui-se que o QAES apresenta evidências de validade baseado na estrutura interna e na relação com outras variáveis, além de índices de precisão adequados. Percebe-se então que a existência de um instrumento que avalie o processo de adaptação ao ensino superior, possibilitando interpretações válidas e precisas, parece ser relevante para o avanço do conhecimento na área. Futuros estudos podem auxiliar na melhor compreensão das questões relacionadas à adaptação acadêmica no ensino superior. É importante investigar a percepção dos docentes sobre a adaptação ao ensino superior e as possíveis ações que são possam ser efetivadas para promover maior adaptação dos alunos.

Dada a importância do tema, sugere-se que as instituições invistam em serviços para auxiliar à adaptação dos estudantes ao ensino superior e tendo como medida o QAES, que pode servir para subsidiá-la. Isto pode ser feito em conjunto com a orientação profissional, tendo como finalidade auxiliar os alunos na construção da carreira e inserção no mercado de trabalho, de forma eficaz.

\section{Referências}

Alliprandim, P. M. Z., \& Souza, D. A. de (2016). A crença de autoeficácia dos formandos de um curso de pedagogia em relação ao exercício profissional. Educação em Análise, 2(1), 215-235. doi:10.5433/1984- 7939.2016v1n2p215

Ambiel, R. A. M., \& Barros, L. O. (2018). Relações entre evasão, satisfação com escolha profissional, renda e adaptação de universitários. Psicologia: Teoria e Prática, 20(2), 254-267 doi:10.5935/1980-6906/psicologia.v20n2p254267

Ambiel, R. A. M., Santos, A. A. A., \& Dalbosco, S. N. P. (2016). Motivos para evasão, vivências acadêmicas e adaptabilidade de carreira em universitários. Psico-RS, 47(4), 288-297. doi:10.15448/1980-8623.2016.4.23872

Araújo, A. M., Almeida, L. S., Ferreira, J. A., Santos, A. A., Noronha, A. P., \& Zanon, C. (2014). Questionário de Aadaptação ao Ensino Superior (QAES): Construção e validação de um novo questionário. Psicologia, Educação e Cultura, 1(1), 131145. doi:10.1037/t59929-000

Audibert, A., \& Teixeira, M. A. P. (2015). Escala de Adaptabilidade de Carreira: Evidências de validade em universitários brasileiros. Revista Brasileira de Orientação Profissional, 16(1), 83-93. Recuperado de http://pepsic.bvsalud.org/pdf/rbop/v16n1/09.p df

Bandura, A. (1993). Perceived self-efficacy in cognitive development and functioning. Educational Psychologist, 28(2), 117-148.

Bardagi, M. P., \& Hutz, C. S. (2011). Eventos estressores no contexto acadêmico: Uma breve revisão da literatura brasileira. Interação Psicológica, 15(1), 111-119. 
doi:10.5380/psi.v15i1.17085

Costa, A. R., Araújo, A. M., \& Almeida, L. S. (2014). Relação entre a percepção da autoeficácia acadêmica e o engagement de estudantes de engenharia. International Journal of Developmental and Educational Psycology, 1(2), 307-314. Recuperado de http://dehesa.unex.es/bitstream/handle/10662/ 2034/0214-

9877_2014_1_2_307.pdf?sequence=1\&isAllo wed $=\mathrm{y}$

Dalbosco, S. N. P. (2018). Adaptação acadêmica no ensino superior: estudos com ingressantes. (Tese de Doutorado, Universidade São Francisco, Campinas, São Paulo). Recuperado de

https://www.usf.edu.br/galeria/getImage/427/ 10206329435389866.pdf

Gamboa, V., Paixão, O., \& Palma, A. I. (2014). Adaptabilidade de carreira e autoeficácia da transição para o trabalho: $\mathrm{O}$ papel da empregabilidade percebida - estudo com estudantes do ensino superior. Revista Portuguesa de Pedagogia, 48(2), 133-156. doi:10.14195/1647-8614_48-2_7

Granado, J. I. F., Santos, A. A. A., Almeida, L. S., Soares, A. P., \& Guisande, M. A. (2005). Integração académica de estudantes universitários: Contributo para adaptação e validação do QVA-r no Brasil. Psicologia e Educação, 4(2), 31-41. Recuperado de https://repositorium.sdum.uminho.pt/bitstream /1822/12089/1/Granado\%2C\%20Santos\%2C $\% 20$ Almeida $\% 2 \mathrm{C} \% 20$ Soares $\% 20 \% 26 \% 20 \mathrm{Gu}$ isande $\% 2 \mathrm{C} \% 202005$.pdf

Hu, L., \& Bentler, P. M. (1999). Cutoff criteria for fit indexes in covariance structure analysis: Conventional criteria versus new alternatives. Structural Equation Modeling: A Multidisciplinary Journal, 6(1), 1-55. doi:10.1080/10705519909540118

Ledesma, R. D., Ferrando, P. J., \& Tosi, J. D. (2019). Uso del análisis factorial exploratorio en RIDEP. Recomendaciones para Autores y Revisores. Revista Iberoamericana de Diagnóstico y Evaluación - e Avaliação Psicológica, 52(3), 173-180. doi:10.21865/RIDEP52.3.13

Levin, J., \& Fox, J. A. (2004). Estatísticas para ciências humanas. São Paulo, SP: Pearson.
Luca, L., Noronha, A. P. P, \& Queluz, F. N. F. R. (2018). Relações entre estratégias de coping e adaptabilidade acadêmica em estudantes universitários. Revista Brasileira de Orientação Profissional, 19(2), 169-176. doi:1026707/1984-7270/2019v19n2p169

Marôco, J. (2014). Análise estatística com o SPSS Statistics. Pêro Pinheiro, Portugal: Report Number.

Mognon, J. F., \& Santos, A. A. A. (2013). Relação entre vivência acadêmica e os indicadores de desenvolvimento de carreira em universitários. Revista Brasileira de Orientação Profissional, 14(2), 227-237. Recuperado de http://pepsic.bvsalud.org/scielo.php?script=sci _issues\&pid=1679-3390\&lng=pt\&nrm=iso

Oliveira, K. L. de, Santos, A. A. A., \& Inácio, A. L. M. (2018). Adaptação Acadêmica e estilos intelectuais no ensino superior. Estudos Interdisciplinares em Psicologia, 9(supl.), 7389. doi:10.5433/2236-6407.2018v9n3suplp73

Ornelas, L. H. B, Díaz-Leal, A. C., Contreras, M. O., Fernández, F. M., \& Pinto, N. S. (2019). La Autoeficacia en el Cuidado de la Salud en la Predicción de la Satisfacción con la Vida. Revista Iberoamericana de Diagnóstico y Evaluación - e Avaliação Psicológica, 52(3), 53-65. doi:10.21865/RIDEP52.3.0

Pasquali, L. (2015). Delineamento de pesquisa em ciência: Volume 2. São Paulo, SP: Vetor.

Polydoro, S. A. J., \& Guerreiro-Casanova, D. C. (2010). Escala de auto-eficácia na formação superior: Construção e estudo de validação. Avaliação Psicológica, 9(2), 267-278. Recuperado de http://pepsic.bvsalud.org/pdf/avp/v9n2/v9n2a 11.pdf

Rosas, J. S. (2013). Búsqueda de ayuda acadêmica, autoeficácia social e acadêmica y emociones de logro en clase en estudiantes universitários. Revista Argentina de Ciencias del Comportamiento, 5(1), 35-41. Recuperado de

https://revistas.unc.edu.ar/index.php/racc/artic le/view/5158/5323

Santos, A. S., Oliveira, C. T. de, \& Dias, A. C. G. (2015). Características das relações dos universitários e seus pares: Implicações na 
adaptação acadêmica. Revista Psicologia:

Teoria e Prática, 17(1), 150-163. doi:10.15348/1980-6906

Savickas, M. L., \& Porfeli, E. J. (2012). Career Adapt-Abilities Scale: Construction, reliability, and measurement equivalence across 13 countries. Journal of Vocational Behavior, $\quad 80(3), \quad 661-673$. doi:10.1016/j.jvb.2012.01.011

Savickas, M. L., Nota, L., Rossier, J., Dauwalder, J. P., Duarte, M. D., Guichard, J. P., Soresi, S., Esbroeck, R. V., \& Von Vianen, A. E. W. (2009). Life designing: Paradigm for career construction in the 21 st century. Journal of Vocational Behavior, 75(3), 239-250. doi:10.1016/j.jvb.2009.04.004

Schreiber, J. B., Nora, A. Stage, F. K., Barlow, E. A. \& King, J. Barlow, E. A. (2006). Reporting structural equation modeling and confirmatory factor analysis results: A review. The Journal of Educational Research, 99(6), 323-337. doi:10.3200/JOER.99.6.323-338

Silva, M. C. R., Ferraz, V. E., \& Oswaldo, Y. C. (2012). Auto-eficácica: Uma avaliação do formato universitário frente à transposição para o mercado de trabalho. Encontro Revista de Psicologia, 15(22), 107-120. Recuperado de http://revista.pgsskroton.com.br/index.php/ren c/article/view/2483/2379

Soares, A. B., Leme, V. B. R., Gomes, G., Penha, A. P., Maia, F. A., Lima, C. A., Valadas, S., Almeida, L. S., \& Araújo, A. M. (2018). Expectativas acadêmicas de estudantes nos primeiros anos do Ensino Superior. Arquivos Brasileiros de Psicologia, 70(1): 206-223. Recuperado de http://repositorio.uportu.pt/jspui/bitstream/113 28/2553/1/15.pdf

Soares, A. B., Seabra, A. M. R. de, \& Gomes, G. (2014). Inteligência, autoeficácia e habilidades sociais em estudantes universitários. Revista Brasileira de Orientação Profissional, 15(1), 85-94. Recuperado de http://pepsic.bvsalud.org/scielo.php?script=sci _issues\&pid=1679-3390\&lng=pt\&nrm=iso

Stajkovi, A. D., Bandura, A., Locke, E. A., Lee, D., \& Sergent, K. (2018). Test of three conceptual models of influence of the big five personality traits and self-efficacy on academic performance: A meta-analytic pathanalysis. Personality and Individual Differences, 120, 238-245. doi:10.1016/j.paid.2017.08.014

Tinto, V. (2007). Research and practice of student retention: what next? Journal of College Student Retention, 8(1), 1-19. Recuperado de https://journals.sagepub.com/doi/pdf/10.2190/ 4YNU-4TMB-22DJ-

AN4W?casa_token=_sHUcPE_nhsAAAAA:I 7S5FcFstfEa3O06u0w9EprmkhWgFcacHTajTzJ9kmKmgFC c4UQcBtlS021vBtea1c6XCvggVDEMg

Tornimbeni, S., Pérez, E., \& Olaz, F. (2008). Introducción a la psicometria. Buenos Aires: Paidós.

Wainer, J., \& Melguizo, T. (2018). Políticas de inclusão no ensino superior: Avaliação do desempenho dos alunos baseado no Enade de 2012 a 2014. Educação e Pesquisa, 44(e162807), 1-15. doi:10.1590/s1517-9702201612162807 